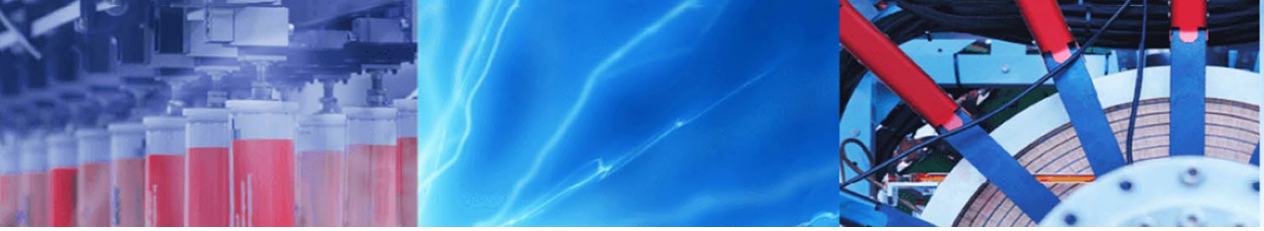

Research Article

\title{
Mathematical modeling of ethylene polymerization over advanced multisite catalysts: an artificial intelligence approach
}

\author{
Saeid Atashrouz ${ }^{1} \cdot$ Mohammad Rahmani $^{1,2}$ [D $\cdot$ Zahra Balzadeh $^{3} \cdot$ Bahram Nasernejad $^{2}$
}

Received: 15 September 2019 / Accepted: 22 January 2020 / Published online: 6 February 2020

(c) Springer Nature Switzerland AG 2020

\begin{abstract}
Recent developments in multisite catalysts based on metallocenes and post-metallocenes attracted the attention of researchers and industrial petrochemical companies due to the production of high-performance polymeric materials which generally are not achievable based on Ziegler-Natta catalysts. In this study, with the aim of predicting the average molecular weight of produced polyethylene and activity of ethylene polymerization using multisite catalysts, robust precise models based on artificial neural networks are developed. The average error for the prediction of the average molecular weight and activity are $3.76 \%$ and $5.89 \%$, respectively. The Leverage method was used to check the reliability of the proposed model and the quality of experimental data which have been used for model development. The results showed that just a few data points are outside of the applicability domain of the developed models, confirming that both developed models and their predictions are statistically correct. Comparison of the artificial neural network models with other artificial intelligence approaches including support vector machine and group method of data handling type neural networks illustrates the better performance and robustness of the proposed models. The results of this study promise that neural networks can be used as reliable models with reasonable accuracy to estimate the performance of ethylene polymerization over this type of new metallocene/post-metallocene multisite catalysts.
\end{abstract}

Keywords Neural networks $\cdot$ Ethylene polymerization $\cdot$ Multisite catalyst $\cdot$ Mathematical modeling $\cdot$ Metallocene

\section{Introduction}

Polyolefin materials like polyethylene and polypropylene have significant properties like excellent mechanical/ thermal properties, fine melt strength and high processability, recycling ability, light weight, high flexibility and good chemical resistance. Such properties result in considering these polymers as affordable raw materials for diverse applications from high-pressure pipes, fuel tanks, advanced heat floor pipes to shopping bags, toys, bottles and car bumpers [1, 2].

The invention of Ziegler-Natta catalysts in 1953 was led to the production of polyethylene and isotactic polypropylene under mild reaction conditions. This success was a revolution in the polyolefin industry which led to Nobel prize for Ziegler and Natta in 1963 [3]. However, due to "uncontrolled" multisite nature of Ziegler-Natta catalysts, precise control over the microstructure of polymers produced by this types of catalyst is not possible. Unlike Ziegler-Natta catalysts, the single site metallocene catalysts, basically consisting of a sandwiched transition metal center between two cyclopentadienyl ligands, benefited by well-known active site structure. Taking this advantage, the precise control over the key architectural characteristics of the polymer such as weight average molecular weight (MW), distribution of molecular weight (MWD)

\footnotetext{
$\triangle$ Mohammad Rahmani, m.rahmani@aut.ac.ir $\mid{ }^{1}$ Chemical Engineering Department, Amirkabir University of Technology, Mahshahr Campus, Mahshahr, Iran. ${ }^{2}$ Chemical Engineering Department, Amirkabir University of Technology, Tehran, Iran. ${ }^{3}$ Polymer Engineering Department, Amirkabir University of Technology, Tehran, Iran.
} 
and short chain branching distribution (SCBD) becomes possible [4-6]. Due to the well-known structure of this single site pre-catalysts, the systematic study of initiation, propagation and termination steps is provided. However, this type of catalysts produces polymers exhibiting narrow molecular weight distribution suffering from poor processability [7]. Furthermore, in order to implement this type of catalyst in industrial applications, it is necessary to immobilize metallocene and post-metallocene catalysts on a suitable support $[7,8]$. Over the recent years, the multisite catalyst technology has been proposed by researchers to immobilize homogeneous active sites on different supports comprising $\mathrm{MgCl}_{2}$, silica, and graphene [7-10]. In this technology generally two or three different types of metallocenes and post-metallocene catalysts are immobilized on one or more preferred kinds of supports $[8,10-14]$. As a consequence, polymers produced using multi component catalysts exhibit multi modal molecular weight distribution [7]. A series of multisite catalysts were developed by Mülhaupt and Co-workers in which bimodal or trimodal polyethylene possessing precisely controlled microstructures are obtained $[8,10-18]$ One of their outstanding results, was the successful development of a polyethylene additive, that caused significant improvement in HDPE mechanical properties. The polymer stiffness, toughness and tensile strength were increased simultaneously, in terms of tensile strength $(+392 \%)$, impact resistance (+ 197\%) and Young's modulus (+ 365\%) [8]. A comprehensive review of the literature on multisite catalysts has been conducted by Stürzel et al. [7].

Although a series of outstanding and useful experimental studies have been conducted in the area of multisite catalyst based ethylene polymerization, modeling the behavior of this type of catalysts has also a great importance from the both industrial and academic point of views, specially scale up issues. In addition, doing many experimental studies to check the different performance of the catalyst in diverse process conditions is many expensive, tedious and extremely time and energy-consuming. In this regard, a predictive approach like artificial intelligence (Al) can be helpful. Over recent years, Al approaches like artificial neural networks (ANN), group method of data handling type neural network systems (GMDH-NN) and least square support vector machines (LSSVM) have widely been used by researchers to model various chemical engineering systems [19-25]. Each one of this approaches benefited by its own advantages. For instance, based on GMDH-NN approach it is possible to obtain an explicit analytical relationship between input and output of the problem [21]. Also, the SVM approach has a different benefit compared to other $\mathrm{Al}$ approaches in which due to implementing of quadratic programming as a convex function only one global solution for the studied system is found $[26,27]$. On the other hand, neural networks have the benefit of fantastic flexibility to model highly non-linear systems. In this regards, in systems with much complexity, it is preferred to implement neural network models.

The aim of the current study is implementing ANN systems to model the average molecular weight of polyethylene obtained by advanced multisite catalyst besides the catalytic system activity. To the best knowledge of authors, to date, no mathematical models based on Al methods have been developed for this purpose. To develop the model, the concentration of pre-catalysts and polymerization time are considered as the input parameters and average molecular weight and activity are considered as the model outputs. The models performance is validated by various statistical functions. Also, the Leverage approach is used to evaluate the reliability of the ANN models and the experimental data. Ultimately, the artificial neural network model performance is compared with other $\mathrm{Al}$ approaches comprising SVM and GMDH to determine the superior model for this purpose.

\section{Theoretical background}

\subsection{Multisite catalysts and multimodal PE}

Generally, polyethylene with low molecular weight has high crystallization rate, low viscosity and high stiffness. While ultrahigh molecular weight polyethylene (UHMWPE) is benefitted by high toughness, much lower crystallization rate and higher melt viscosity. The high viscosity of this macromolecules originates from their highly entangled chains compared to medium molecular weight highdensity polyethylene (HDPE). Figure 1 illustrates bimodal molecular weight distribution (MWD) for polyethylene in which UHMWPE with short chain branches plays the role of tie molecule and links polyethylene crystal lamellae. In this regard, the existence of UHMWPE in polyethylene structure causes the significant promotion of fatigue resistance, environmental stress crack resistance (ESCR), abrasion resistance and impact strength.

This type of MWD is necessary to produce sustainable lightweight materials possessing high resistance like high performance pipes (damage resistance) and under pressure tanks [7]. Development of cascade reactor technology to obtain in reactor blend of HDPE and UHMWPE compounds resulted in commercial manufacturing of $P E$ pipes with enhanced performance. However, the stateof-the-art of production bimodal PE for pipe applications, has shown that maximum incorporation of UHMWPE in the product which is applicable based on cascade reactor technology is less than $3 \mathrm{wt} \%[7,28-34]$. While in the case 
Fig. 1 Bimodal MWD of polyethylene and its schematic structure [7]
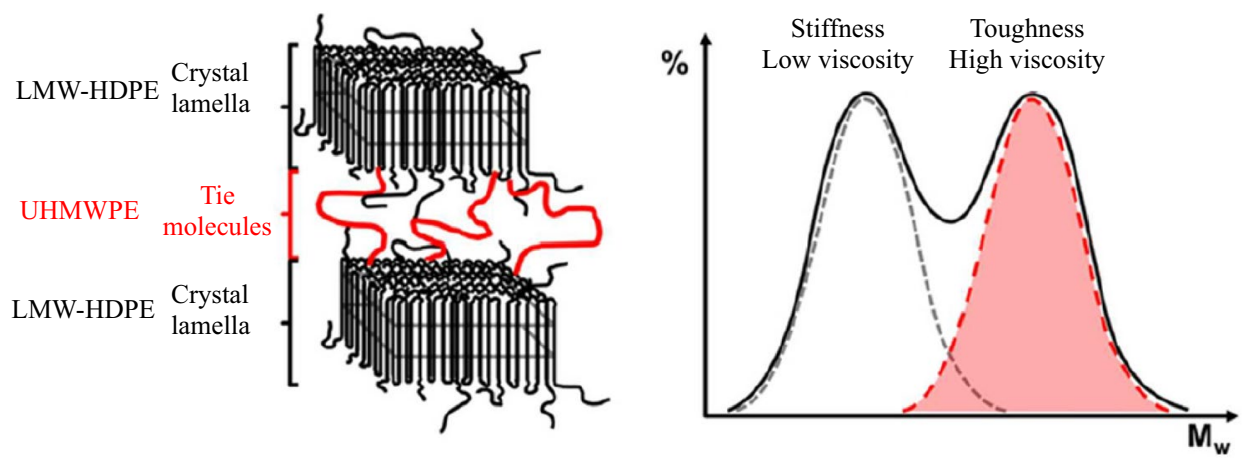

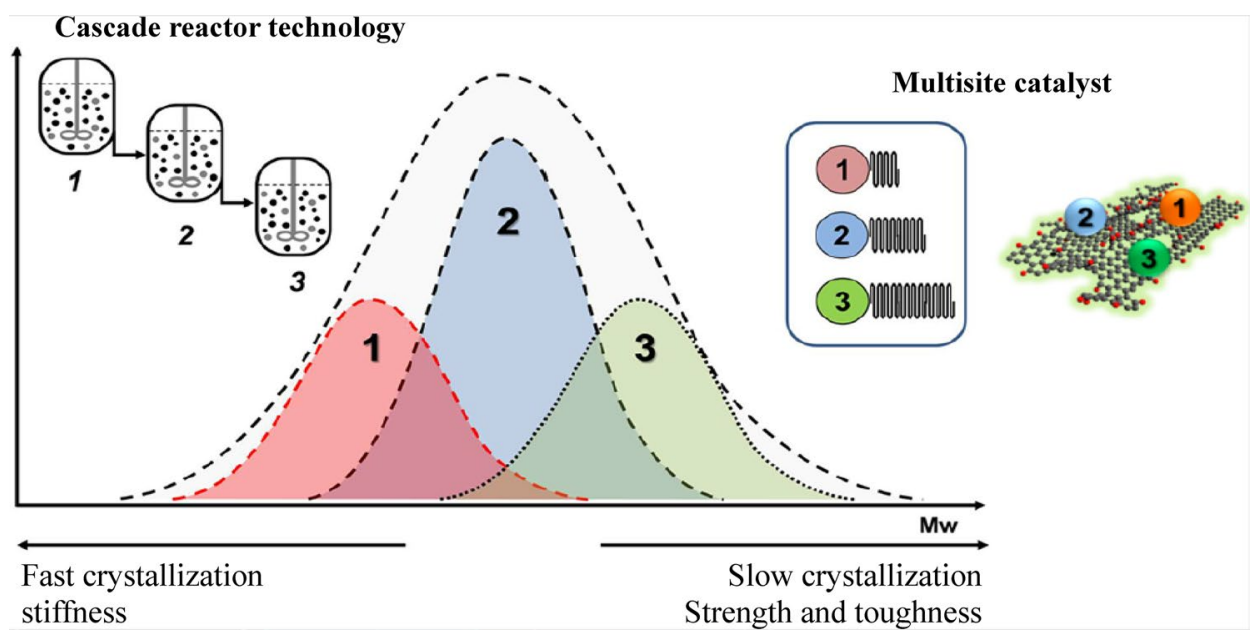

Fig. 2 Achievement of Multimodal MWD for polyethylene based on cascade reactor technology (above left) and multisite catalyst technology in single reactor (above right) [7]

of advanced multisite catalyst technology, the immobilization of various single site catalysts (metallocene/postmetallocene pre-catalysts) on a suitable support, results in the incorporation of almost $12 \mathrm{wt} \%$ of UHMWPE in HDPE. In this regard based on multisite catalysts, development of PE commodities with higher performances (improved mechanical properties) will be provided.

As can be observed in Fig. 2, according to the cascade reactor technology (in reactor blending of UHMWPE and HDPE) and using Ziegler-Natta catalysts, multimodal MWD can be achieved. Generally, in this technology the desired MWD is obtained by altering the process conditions, hydrogen and comonomer concentrations in three reactors. However, another technology which is based on multisite catalysts is performed in just one reactor and has more benefits compared to cascade reactor technology. In this type of catalyst, each single site catalyst polymerized ethylene to polyethylene with a certain range of molecular weight. Thus, one site can produce UHMWPE and another can be used for the production of HDPE and in this regard the required MWD is achieved in a single reactor. According to the report of the Univation Technologies company, a value more than $\$ 35$ million will be the investment savings for a single UNIPOL ${ }^{\text {TM }}$ PE Process reactor with $300 \mathrm{kta}$ production in comparison with cascade reactor technology. Also, about $35 \%$ savings in operating costs is another advantage of this technology compared to cascaded reactor technology. Consequently, not only it is possible to produce polyethylene with promoted properties but also lower production costs are estimated using the multisite catalyst technology in a single reactor.

\subsection{Artificial neural networks}

As a general schematic of ANNs, they are a replication of brain process to analyze a phenomenon. ANNs consist of interconnected group of neurons in multiple layers making the backbone of ANNs. The information of the studied problem is introduced to the network by the first layer which is called "input layer" [35]. In addition, hidden layers which enrich the network for learning the system, have the duty of connecting the input and output layers. From the theoretical point of view, only one hidden layer with 
appropriate neurons has the ability to learn any behavior of a system [36]. The relation between neuron output and the network is provided by a mathematic function called the transfer function. The most conventional transfer function for prediction of phenomena are the linear, sigmoid and Tansig transfer functions which are easy to differentiate to use in a learning scheme such as back propagation (BP) [37-39]. This transfer functions have following relations:

Linear transferfunction, $\mathrm{g}(\mathrm{x})=\mathrm{x}$

Sigmoid transfer function, $\quad g(x)=\frac{1}{1+e^{-x}}$

Tansig transfer function, $\quad g(x)=\frac{e^{x}-e^{-x}}{e^{x}+e^{-x}}$

For a network with Tansig transfer function for one hidden layer the model output takes the following form:

$y=\operatorname{Linear}\left(W_{2} \times\left(W_{1} \times g(x)_{\text {Tansig }}+B_{1}\right)+B_{2}\right)$

where $W$ and $B$ represents weights and biases respectively.

Generally, the Levenberg-Marquardt (LM) algorithm $[40,41]$ is implemented to optimize weights and biases of the network. In most cases ANN-LM models does not involve local minimums and even with an inappropriate initial guess the global minimum is obtained [42]. In this regard we used this algorithm for optimization of the ANN model. Since the concept of neural networks has been described well in literature, the authors ignored detailed description of this approach. The interested readers is referred to read refs. $[43,44]$ for more information.

\subsection{Group method of data handling type neural networks}

Following the Darwinism's theory, Ivakhneko proposed the group method of data handling (GMDH) technic [45, 46]. In sharp contrast to ANNs in which a group of weights and biases are generated as the output of the model, in the GMDH type neural networks (GMDH-NN) a group of simple polynomials are generated as the final structure of the model. In the GMDH-NN approach the grand correlation polynomial used to model a system takes the following form:

$y=a+\sum_{i=1}^{M} \sum_{j=1}^{M} \ldots \sum_{k=1}^{M} b_{i j \ldots k} x_{i}^{n} x_{j}^{n} \ldots x_{k}^{n}$

where $a$ and $b$ are model constants and $M$ stands for the number of independent variables. Detailed information of step by step modeling procedure of GMDH-NN can be find elsewhere $[21,23,47]$.

\subsection{Least square support vector machine (LSSVM)}

The SVM is generally implemented for applications including pattern recognition, regression analysis and classification. LSSVM is an improved version of SVM which has been proposed to reduce the complexity of SVM approach [48, 49] with following cost function (CF):

$C F=\frac{1}{2} w^{T} w+\frac{1}{2} \gamma \sum_{k=1}^{N} e_{k}^{2}$

where $\gamma$ is an adjustable parameter preventing overfitting problem, $w^{T}$ is the transposed output layer and $e_{k}$ stands for variable error of train data. The following equality constraint is subjected to the cost function:

$y_{k}=w^{T} \varphi\left(x_{k}\right)+b+e_{k}$

where $b$ is the bias vector and $\varphi\left(x_{k}\right)$ is the kernel function. The radial basis function (RBF) was used in the present study as the kernel function of LSSVM model due to its excellent performance for nonlinear systems. The RBF has following form:

$\varphi\left(x, x_{k}\right)=\exp \left(\frac{-\left\|x_{k}-x\right\|^{2}}{\sigma^{2}}\right)$

where $\sigma^{2}$ is the tuning parameter of kernel function. In this regard, there are two tuning parameter for LSSVM comprising $\sigma^{2}$ and $\gamma$ which should be determined. In the present study we used coupled simulated annealing (CSA) approach [50] to achieve this purpose. The implemented algorithm to optimize the LSSVM-CSA model is shown in Fig. 3. More detailed information related to the SVM and LSSVM can be found in the previous studies [20, 48, 51-55].

\section{Model development and evaluation}

In this study, the experimental data which have been reported by Stürzel et al. [13] were used for model development. The authors developed three-site catalyst, immobilized on functionalized graphene in which CrBIP (see Fig. 4 for full name of pre-catalysts) pre-catalyst produces polyethylene wax with high crystallization rate, $\mathrm{CrQCp}$ forms UHMWPE, and FeBIP or ZrCp are as the active site responsible for HDPE production with variable intermediate molar mass. The mentioned active sites are shown in Fig. 4. Also, experimental data which have been implemented for the model training are tabulated in Table 1.

Since the aim is to model average molecular weight and activity, these parameters are supposed to be a function of 
Fig. 3 Implemented algorithm to develop LSSVM-CSA model

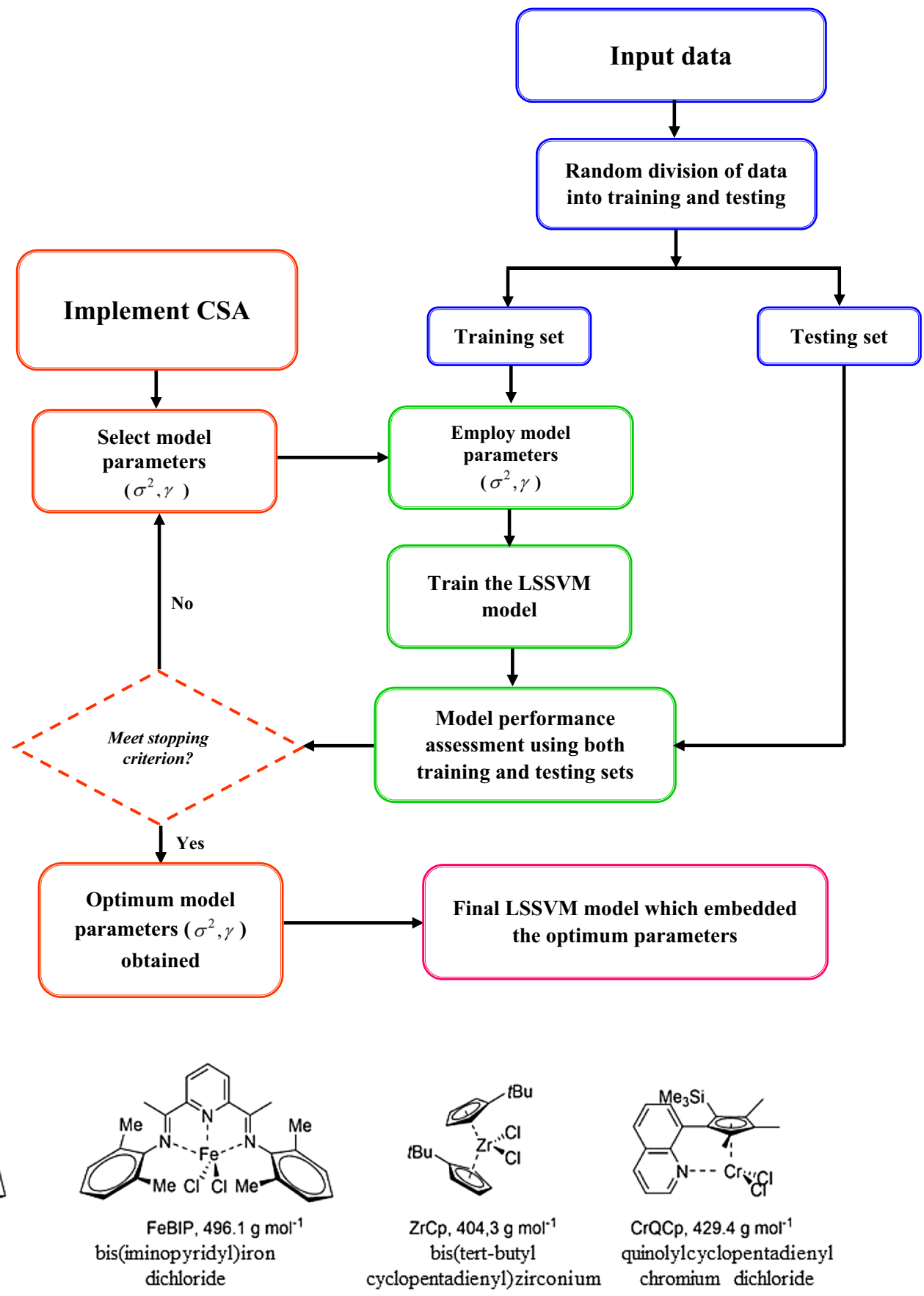

Fig. 4 Types of the single-site pre-catalysts used in multisite catalyst by Stürzel et al. [13] to produce trimodal polyethylene

the concentration of pre-catalyst and time of polymerization $\left(t_{p}\right)$ as below:

$\mathrm{Mw}=f\left(\mathrm{CrBIP}, \mathrm{FeBIP}, \mathrm{ZrCp}, \mathrm{CrQCp}, t_{p}\right)$

Activity $=f\left(C r B I P\right.$, FeBIP, ZrCp, CrQCp, $\left.t_{p}\right)$
In this regard, two neural networks were developed separately to predict $M_{w}$ and activity. Figure 5 illustrates a schematic of the proposed ANNs along with inputs and output to the model for the prediction of Mw. The same schematic can be drawn for the second neural network which predicts activity.

In order to develop the ANNs, the experimental data were randomly divided into three groups. The main part 
Table 1 Experimental data used in this study to develop the models

\begin{tabular}{lllllllc}
\hline Entry & $\begin{array}{l}\text { CrBIP } \\
\left.(\mu \mathrm{mol} \mathrm{L})^{-1}\right)\end{array}$ & $\begin{array}{l}\text { FeBIP } \\
\left.(\mu \mathrm{mol} \mathrm{L})^{-1}\right)\end{array}$ & $\begin{array}{l}\mathrm{ZrCP} \\
(\mu \mathrm{mol} \mathrm{L})\end{array}$ & $\begin{array}{l}\text { CrQCP } \\
\left.(\mu \mathrm{mol} \mathrm{L})^{-1}\right)\end{array}$ & $\begin{array}{l}\mathrm{t}_{\mathrm{p}} \\
(\mathrm{min})\end{array}$ & $\begin{array}{c}\text { Activity } \\
\left(\mathrm{g} \mathrm{mmol}^{-1} \mathrm{~h}^{-1}\right)\end{array}$ & $\begin{array}{l}\mathrm{Mw} \\
\left(\mathrm{Kg} \mathrm{mol}^{-1}\right)\end{array}$ \\
\hline 1 & 0 & 2 & 0 & 0 & 120 & 26,200 & 270 \\
2 & 6.3 & 2 & 0 & 0.8 & 120 & 9300 & 230 \\
3 & 6.3 & 1.3 & 0 & 0.8 & 120 & 15,300 & 650 \\
4 & 6.3 & 0.7 & 0 & 0.8 & 120 & 16,900 & 400 \\
5 & 6.3 & 1 & 0 & 0.8 & 120 & 14,900 & 470 \\
6 & 6.3 & 1 & 0 & 1.6 & 120 & 16,200 & 570 \\
7 & 6.3 & 1 & 0 & 3.2 & 120 & 14,400 & 1125 \\
8 & 12.6 & 2.6 & 0 & 3.2 & 240 & 3100 & 660 \\
9 & 7.9 & 1.7 & 0 & 1.9 & 180 & 5000 & 780 \\
10 & 6.3 & 1.3 & 0 & 1.6 & 110 & 6800 & 570 \\
11 & 0 & 0 & 24.7 & 0 & 30 & 4400 & 1600 \\
12 & 9.2 & 0 & 2.3 & 4.7 & 30 & 12,800 & 1750 \\
13 & 9.2 & 0 & 4.9 & 4.7 & 30 & 12,400 & 1240 \\
14 & 9.2 & 0 & 9.9 & 4.7 & 30 & 12,000 & 830 \\
15 & 9.2 & 0 & 9.9 & 2.3 & 30 & 4400 & 640 \\
16 & 9.2 & 0 & 9.9 & 1.6 & 30 & 7800 & 610 \\
17 & 18.5 & 0 & 2.3 & 4.7 & 30 & 10,100 & 710 \\
18 & 18.5 & 0 & 4.9 & 4.7 & 30 & 11,000 & 680 \\
19 & 18.5 & 0 & 9.9 & 4.7 & 30 & 13,300 & 450 \\
20 & 18.5 & 0 & 9.9 & 2.3 & 30 & 7300 & 600 \\
21 & 18.5 & 0 & 9.9 & 1.6 & 30 & 9200 & 400 \\
\hline & & & & & & &
\end{tabular}

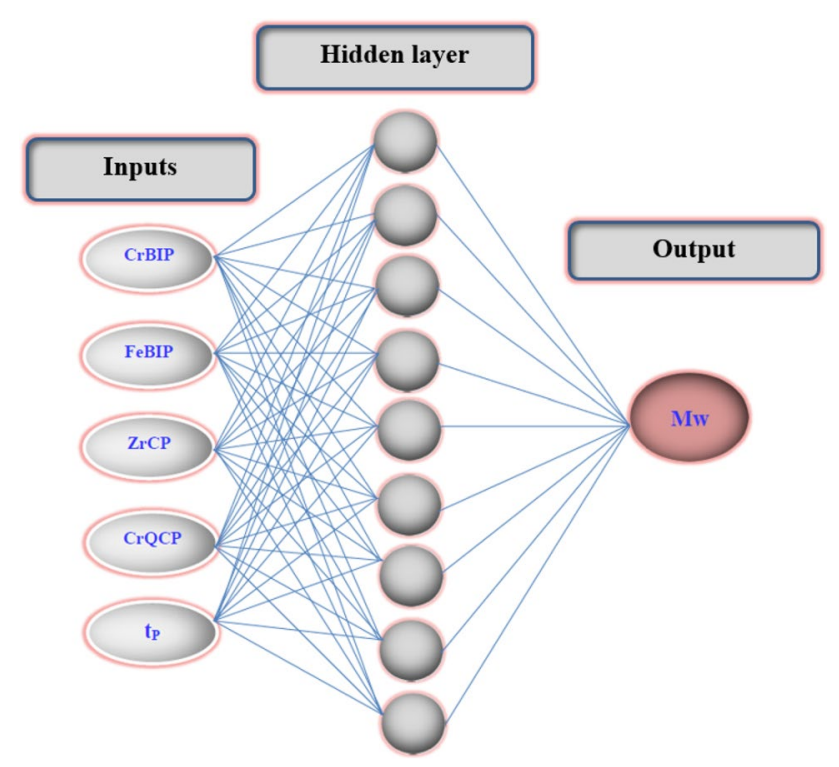

Fig. 5 A schematic of the proposed neural network system to pre$\operatorname{dict} \mathrm{Mw}$

which forms $60 \%$ of the database was used for training of the model and the remained data was equally distributed to validate and test of the model. It must be emphasized that this is a practical approach to employ about $60-70 \%$ of the database to train the model and assigning rest of data for training and testing $[47,56,57]$. Also, to avoid complexity in ANN models, one hidden layer was considered and the number of neurons was changed to find optimum structures. The optimized ANN structures to predict $M w$ and activity are shown in Tables 2 and 3 respectively.

\subsection{Performance evaluation}

To check the performance of an artificial intelligence model like ANN, it is necessary to implement different statistical functions including average relative deviation percentage (ARD\%), regression coefficient $\left(R^{2}\right)$, Root of mean square error (RMSE), average absolute relative deviation percentage (AARD\%) and standard deviation (SD) which their mathematical functions are described below:

1. $A R D \%$ this function is used with the aim of investigating relative deviation between model results and their correspondence experimental data:

$$
A R D(\%)=\frac{100}{N} \sum_{i=1}^{N} \frac{p^{c a l c}-p^{\exp t}}{P \exp t}
$$


Table 2 Architecture of the optimized ANN for prediction of Mw

\begin{tabular}{|c|c|c|c|c|c|c|}
\hline \multirow[t]{2}{*}{ Network type } & \multirow[t]{2}{*}{ Training algorithm } & \multirow{2}{*}{$\begin{array}{l}\text { Input layer } \\
\text { No. of neurons }\end{array}$} & \multicolumn{2}{|l|}{ Hidden layer } & \multicolumn{2}{|l|}{ Output layer } \\
\hline & & & No. of neurons & Activation function & No. of neurons & Activation function \\
\hline $\begin{array}{l}\text { FFBP-ANN (newff } \\
\text { MATLAB function) }\end{array}$ & $\begin{array}{l}\text { train/m MATLAB } \\
\text { function }\end{array}$ & 5 & 8 & $\begin{array}{l}\text { tansig MATLAB func- } \\
\text { tion }\end{array}$ & 1 & $\begin{array}{l}\text { Linear (purelin } \\
\text { MATLAB func- } \\
\text { tion) }\end{array}$ \\
\hline
\end{tabular}

Table 3 Architecture of the optimized ANN for prediction of activity

\begin{tabular}{|c|c|c|c|c|c|c|}
\hline \multirow[t]{2}{*}{ Network type } & \multirow[t]{2}{*}{ Training algorithm } & \multirow{2}{*}{$\begin{array}{l}\text { Input layer } \\
\text { No. of neurons }\end{array}$} & \multicolumn{2}{|l|}{ Hidden layer } & \multicolumn{2}{|l|}{ Output layer } \\
\hline & & & No. of neurons & Activation function & No. of neurons & Activation function \\
\hline $\begin{array}{l}\text { FFBP-ANN (newff } \\
\text { MATLAB function) }\end{array}$ & $\begin{array}{l}\text { train/m MATLAB } \\
\text { function }\end{array}$ & 5 & 8 & $\begin{array}{l}\text { tansig MATLAB func- } \\
\text { tion }\end{array}$ & 1 & $\begin{array}{l}\text { Linear (purelin } \\
\text { MATLAB func- } \\
\text { tion) }\end{array}$ \\
\hline
\end{tabular}

where $N$ denotes the number of data points and $P$ is the studied parameter like molecular weight or activity. In addition, the superscripts "calc" and "expt" stand for the data calculated by the models and experimental data, respectively. It should be noted that a positive $\mathrm{ARD}(\%)$ value reflects the model overestimation, while a negative value reflects the underestimation of the model.

2. $\mathrm{AARD}(\%)$ :

$\operatorname{AARD}(\%)=\frac{100}{N} \sum_{i=1}^{N}\left|\frac{P^{c a l c}-P^{\exp t}}{p^{\exp t}}\right|$

As a consequence, smaller AARD(\%) is more desirable.

3. RMSE This function reflects data dispersion around zero error line:

$R M S E=\sqrt{\frac{1}{N} \sum_{i=1}^{N}(\text { Pcalc }-P \exp t)^{2}}$

4. SD Since this function also illustrates a criterion of data dispersion, error scattering reduces when SD is smaller.

$S D=\sqrt{\frac{1}{N-1} \sum_{i=1}^{N}\left(\frac{\text { pcalc }- \text { Pexpt }}{P^{\text {expt }}}\right)^{2}}$

5. $R^{2}$ The mathematical form of this function is as below:

$R^{2}=1-\left[\frac{\sum_{i=1}^{N}\left(P^{\text {calc }}-P^{\text {expt }}\right)^{2}}{\sum_{i=1}^{N}\left(P^{\text {expt }}-\bar{P}\right)^{2}}\right]$

where $\bar{P}$ stands for the average of experimental data. Values of $R^{2}$ close to 1 demonstrates good performance for a model.

\subsection{Investigating applicability domain of the proposed models}

In addition to error functions which were considered in the previous section, determining of applicability domain has also a great importance to evaluate the model performance. The Leverage approach as a popular method has been used in a number of researches for the purpose of outlier detection and to identify applicability domain of the models [20, 27, 42, 58-60].

Hat matrix and standardized residuals are the main concepts of Leverage approach [61] which are defined in Eqs. (16) and (17) respectively:

$H=X\left(X^{t} X\right)^{-1} X^{t}$

$R_{i}=\frac{e_{i}}{\sqrt{M S E\left(1-H_{i i}\right)}}$

In Eq. (16), $X$ represents a $(i \times j)$ dimension matrix in which $i$ and $j$ are the number of data points and input variables respectively, and $t$ is the transpose multiplier. It must be stressed that Hat values are in fact the diagonal elements of the $H$ matrix (Leverages).

In Eq. (17) $e_{i}$ refers to the deviation of model calculated data and its corresponding ith experimental data, MSE stands for the mean square of error for the proposed model and $H_{i i}$ is the Hat index for the data point $i$ (Leverage). To visualize and identify the suspected data or outliers, the Williams plot was used. In such figure commonly standardized residuals are plotted versus Hat values. Also a parameter called warning Leverage $\left(H^{*}\right)$ is defined in Leverage method as below: 


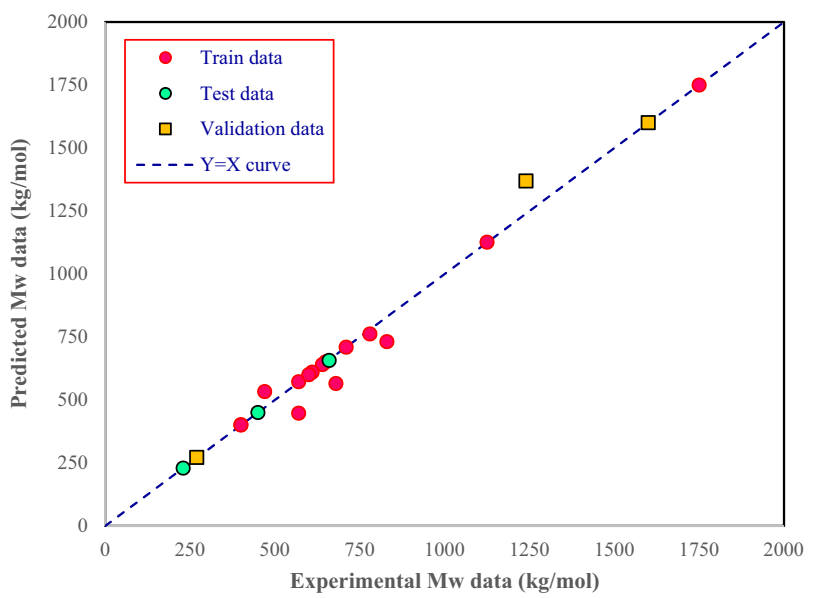

(A)

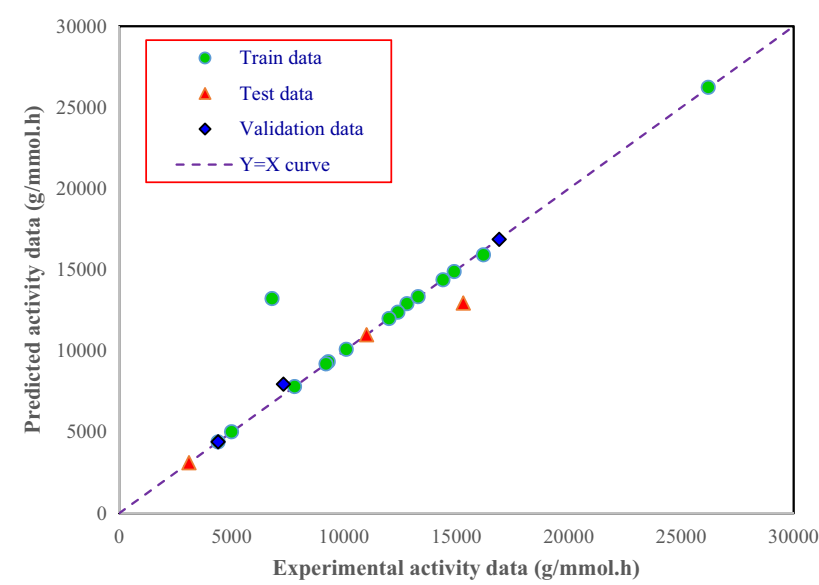

(B)

Fig. 6 Comparison between predicted data by the proposed models and experimental data for: $\mathbf{a}$ Mw and $\mathbf{b}$ Activity

$H^{*}=\frac{3(p+1)}{n}$

where $\mathrm{p}$ and $\mathrm{n}$ demonstrate the number of input parameters and data points, respectively.

Considering Leverage approach to judge statistically validation of a developed model, majority of hat index of data points should be in the range of $0 \leq H \leq H^{*}$ and $-3 \leq R \leq 3[53,62]$. Those group of data which are in the range of $H \geq H^{*}$ and $-3 \leq R \leq 3$ are considered as "Good High Leverage" which albeit are determined to be out of the applicability domain of the model but their standardized residuals are yet acceptable. On the other hand "suspected data" are data points with $R \leq-3$ or $R \geq 3$ which have large uncertainty compared to other data and locate in the outlier domain of the model.
Table 4 Statistical parameters of the proposed ANN models for estimation of Mw and activity

\begin{tabular}{|c|c|c|}
\hline Statistical parameters & $\begin{array}{l}\text { Value for Mw pre- } \\
\text { diction }\end{array}$ & $\begin{array}{l}\text { Value for } \\
\text { activity pre- } \\
\text { diction }\end{array}$ \\
\hline \multicolumn{3}{|l|}{ Training set } \\
\hline AARD $\%$ & 4.48 & 6.58 \\
\hline ARD\% & -2.5 & 6.31 \\
\hline RMSE & 0.015 & 0.011 \\
\hline SD & $3.91 \mathrm{E}-05$ & $1.25 \mathrm{E}-06$ \\
\hline $\mathrm{R}^{2}$ & 0.99 & 1 \\
\hline Number of data points & 15 & 15 \\
\hline \multicolumn{3}{|l|}{ Validation set } \\
\hline AARD $\%$ & 3.66 & 3.06 \\
\hline ARD\% & 3.66 & 2.97 \\
\hline RMSE & 74.31 & 380.32 \\
\hline SD & 0.073 & 0.063 \\
\hline $\mathrm{R}^{2}$ & 0.60 & 0.99 \\
\hline Number of data points & 3 & 3 \\
\hline \multicolumn{3}{|l|}{ Test set } \\
\hline AARD\% & 0.25 & 5.23 \\
\hline ARD\% & -0.25 & -4.98 \\
\hline RMSE & 0.069 & 0.35 \\
\hline SD & 0.0001 & $3.98 \mathrm{E}-05$ \\
\hline$R^{2}$ & 0.99 & 1 \\
\hline Number of data points & 3 & 3 \\
\hline \multicolumn{3}{|l|}{ Total set } \\
\hline AARD $\%$ & 3.76 & 5.89 \\
\hline ARD\% & -1.33 & 4.22 \\
\hline RMSE & 0.026 & 0.009 \\
\hline SD & $6 \mathrm{E}-05$ & $1.08 \mathrm{E}-06$ \\
\hline $\mathrm{R}^{2}$ & 0.99 & 0.99 \\
\hline Number of data points & 21 & 21 \\
\hline
\end{tabular}

\section{Results and discussion}

As mentioned in previous sections, two ANN models were developed to predict $\mathrm{Mw}$ of the produced polymer and activity of the catalyst. A comparison between model results for both studied parameters (including average molecular weight and activity) and their correspondence experimental data is shown in Fig. 6 . As seen, must data points are close to the diagonal line for both models illustrating reasonable conformity between calculated data by the proposed ANN models and experimental data. One of the main problems in ANN models is the "overfitting" issue in which the model has a good performance for train data while its performance for test data leads to wrong results. Table 4 illustrates the values of statistical parameters related to both models for train, test, validation and total data set. According to this results, the performance of both of the models for train, test, and validation is 
Fig. 7 Effect of CrBIP/CrQCP molar ratio on Mw and activity $\left(t_{p}=120 \mathrm{~min}, \mathrm{ZrCP}=0\right.$, $\mathrm{FeBIP}=0$ )

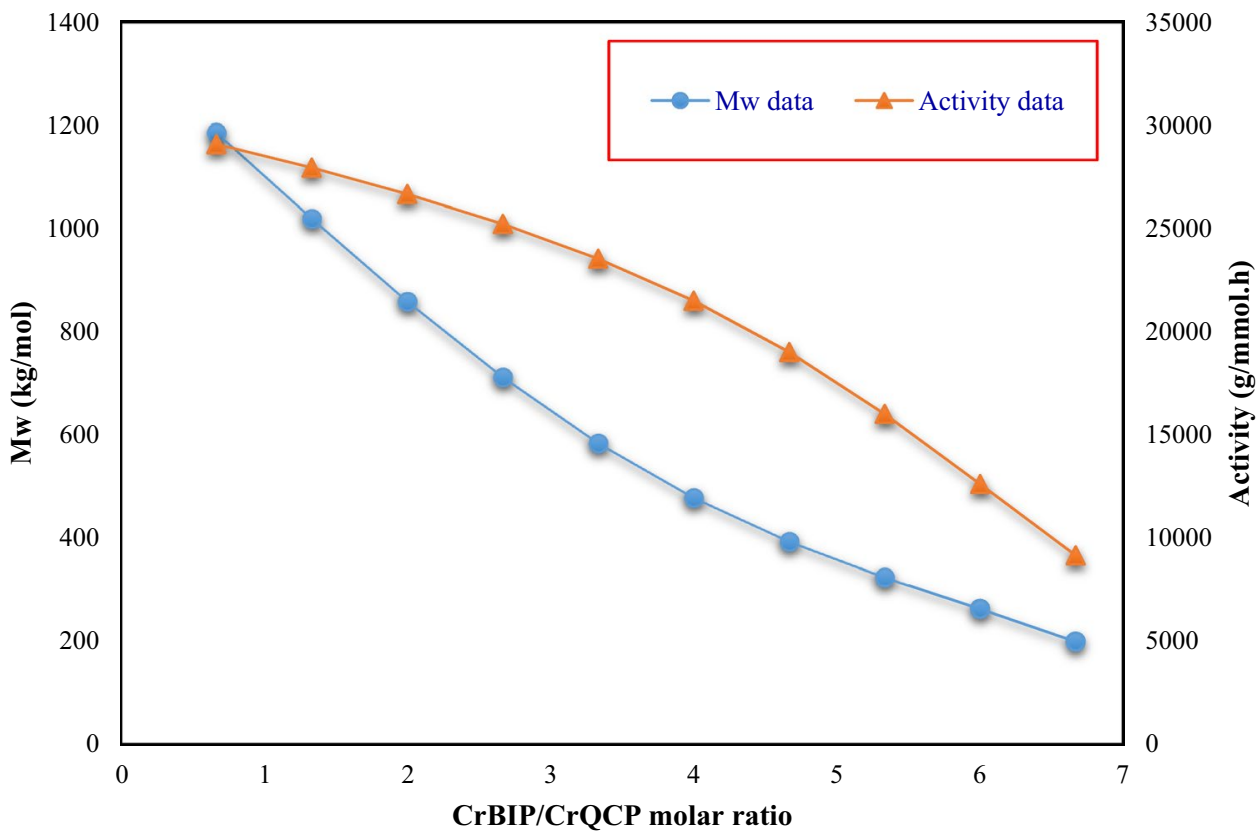

acceptable which clearly demonstrates that the models are not involved with overfitting issue.

In a valuable series of experimental studies by Mülhaupt et al. [8-10, 12, 13, 15-18, 34, 63], various multisite catalysts based on pre-catalysts in Fig. 4 have been developed which interesting results have been obtained in their experiments and observations. As one of their important and practical results, a combination of CrBIP and CrQCP on a single support can lead to a bimodal polyethylene which can be used as an additive to significantly promote HDPE mechanical properties. In this type of catalyst, CrQCP is used to produce long-chain polyethylene (i.e. UHMWPE) which is a reinforcement component for HDPE. However, the addition of UHMWPE to HDPE is a challenge due to the severe entanglement of UHMWPE and consequently high melt viscosity. This makes mixing of UHMWPE with HDPE to be impossible at high UHMWPE portions. So the CrBIP pre-catalyst is used in the multisite catalyst to produce low molecular weight polyethylene (LMWPE) to create nano-phase separation between UHMWPE chains and consequently fantastic improvement of processability is provided.

Figure 7 illustrates the effect of $\mathrm{CrBIP} / \mathrm{CrQCP}$ molar ratio on Mw of polyethylene and also the catalytic system activity. For both curves, increasing the ratio of $\mathrm{CrBIP} / \mathrm{CrQCP}$ leads to the decrement of the target properties. This means for a constant CrQCP concentration, as the concentration of CrBIP active site on support surface increases, not only polymers with shorter chains are produced but also the activity of catalyst will be suppressed. The CrQCP pre-catalyst is a half-sandwich (Fig. 4) chromium-based catalyst with chelating $\mathrm{N}$-donor cyclopentadienyl ligand which makes it a superior catalyst to produce ultrahigh molecular weight polyethylene [64]. Generally, the average molecular weight of the resulting $P E$ based of this precatalyst lies in the range $2 \times 10^{5}-10^{6} \mathrm{~g} / \mathrm{mol}$ for homogeneous polymerization, whereas higher molecular weight ranges are achieved when the pre-catalyst is immobilized on a solid support or when a modifier is used [65]. On the other hand, based on the reported data by Stürzel et al. [13] polymers produced on CrBIP pre-catalyst have low molecular weight. Thus, the predicted trend in Fig. 7 for Mw seems to be rational.

Also, since generally CrQCP has a more activity for ethylene polymerization than CrBIP [13], it is expected that increasing the molar ratio of $\mathrm{CrBIP} / \mathrm{CrQCP}$ should be probably accompanied with a decrease in total activity of multisite catalyst. Such trend is observed in Fig. 7 for the model results.

The Williams plot was implemented to evaluate the reliability of the proposed ANN models, which the results can be seen in Fig. 8. As can be seen for the developed models most of the data points are in the range $-3 \leq R \leq 3$, confirming their reliability and validity from statistical point of view. It must be emphasized that due to the fact that four data points in Fig. 8.A are suspected data, the method recommends that more cautions should be considered when using this model. Also, it is worth noting that no data points have $H \geq H^{*}$ and thus there is no out of Leverage data.

It is worthful to note that when experimental data base for model development is small (e.g. costly and scarce experimental data for studied catalysts in this study) model validation tools become more important. In this regard, 
Fig. 8 Williams plot for the proposed ANN models to identify applicability domain: a related to model for $\mathrm{Mw}, \mathbf{b}$ related to model for activity

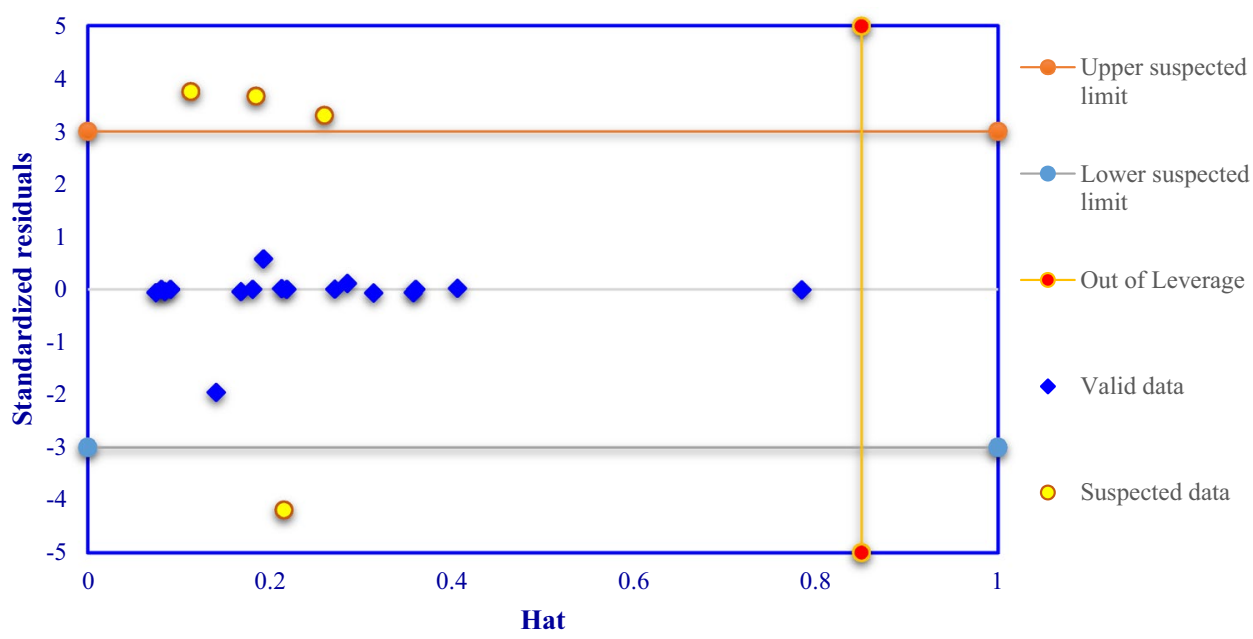

(A)

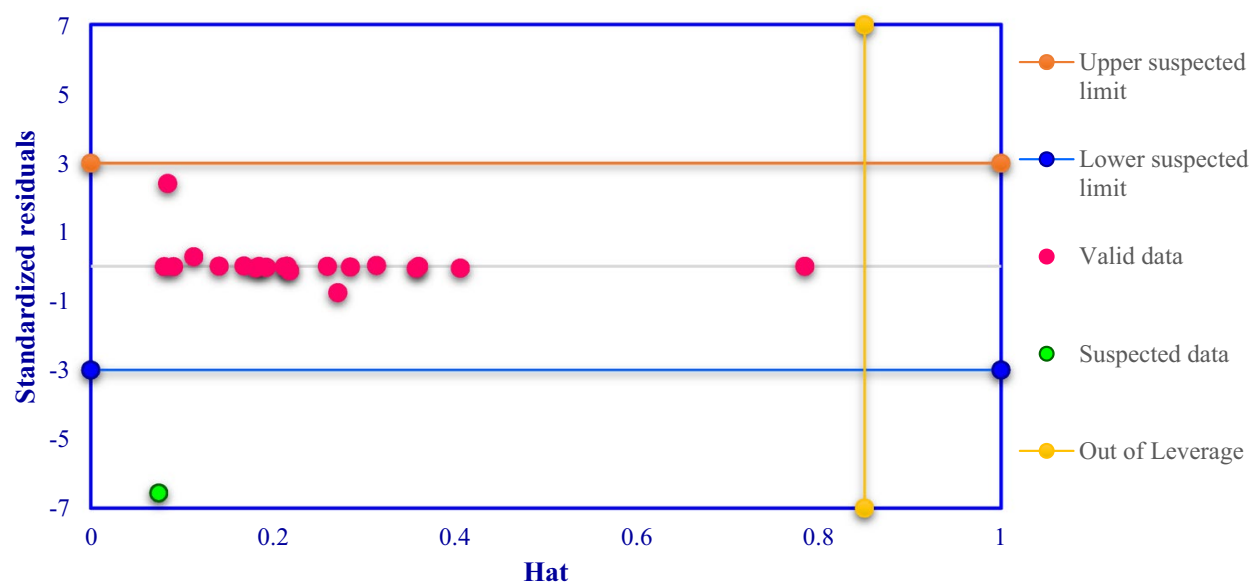

(B)

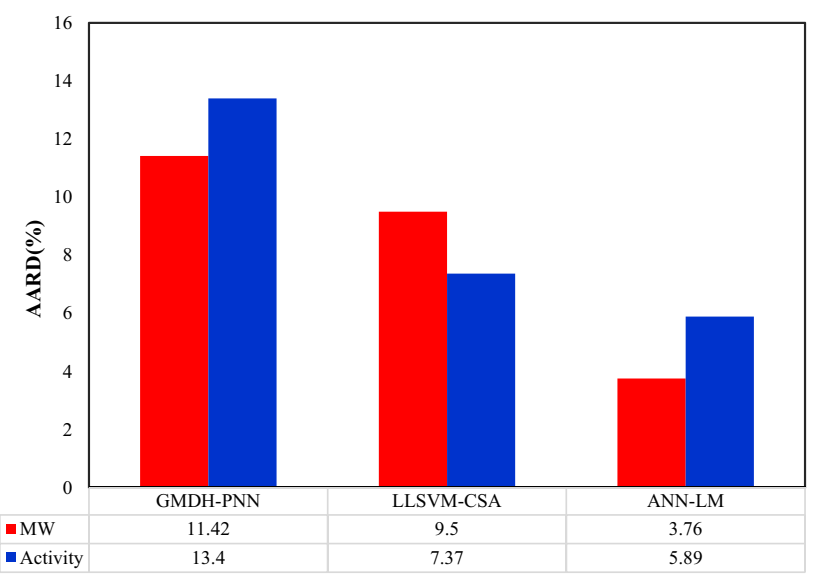

Fig. 9 Comparison of the ANN models with other Al approaches the results of statistical functions to evaluate model accuracy and performance as well as Leverage approach to investigate applicability domain of the proposed models and outlier detection, confirmed the validity of the model. Other examples can be also found in literature which have used small data set for model development. For instance, Yildiz and Uzun [66] have developed a neural network to predict gas storage capacity of metal organic frameworks based on 13 experimental data. Although some statistical functions have been used for model validation, other statistical approaches to check applicability domain of the model have not been used in their study. Furthermore, AANs have been implemented by Anantawaraskul et al. [67] to determine the operating conditions needed to produce polyethylene with a specified microstructure based on 43 experimental data points. However, in their study neither statistical functions nor applicability domain approaches have been considered. But their cross-plot

\section{SN Applied Sciences}


Table 5 The optimized structures of GMDH-PNN models for MW and activity prediction

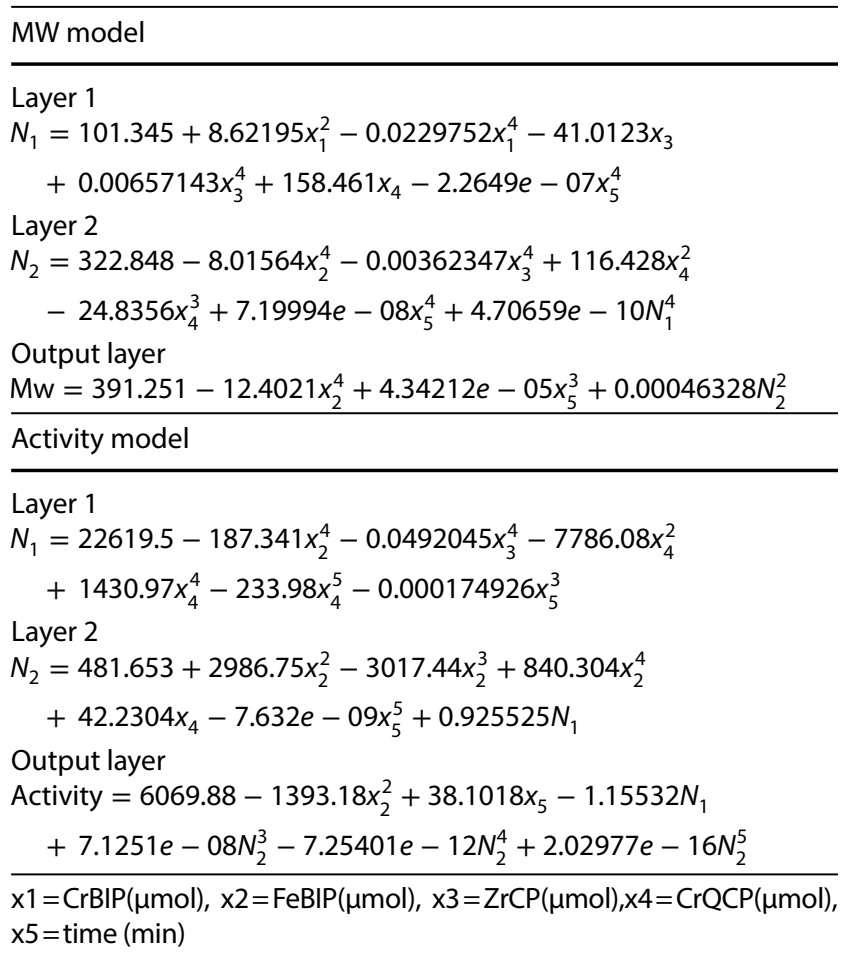

between experimental data and model results exhibits reasonable performance of the developed model.

Finally, to compare ANN-LM with other Al approaches, LSSVM-CSA and GMDH-NN models were considered and their AARD(\%) to predict MW and activity are shown in Fig. 9. As seen, for both properties the ANN-LM accuracy is more than others, illustrating the more flexibility of the ANN-LN model for the mentioned problem. Furthermore, the optimized structures of GMDH-NN models for MW and activity prediction are tabulated in Table 5 . Although the AARD(\%) of GMDH-NN models are more than ANN-LM and LSSVM-CSA, the main distinguished characteristic of GMDH-NN is obtaining explicit analytic relations as the final mathematical structure of the model which makes the model simpler and more applicable than other Al approaches. However, it must be noted that this superiority is acceptable when the accuracy is also in a reasonable range.

\section{Conclusion}

Many valuable experimental studies have been conducted in literature related to multisite catalysts based on metallocene and post-metallocene pre-catalysts. However, the present study deals with a new challenge for advanced multisite catalysts to mathematically model and predict the Mw of the resulted polyethylene and also activity of ethylene polymerization catalysts. Two separate ANN models were developed according to the limited suitable experimental data. According to the results of the models, the AARD $\%$ for prediction of average molecular weight and activity were $3.76 \%$ and $5.89 \%$, respectively. Although statistical functions as well as Leverage approach showed that proposed models in present study were reliable, but in order to develop a comprehensive model, experimental measurements of effect of temperature, pressure, pre-catalyst concentration, hydrogen response, co-catalyst concentration, co-monomer concertation and polymerization time on resulted polymer properties should be conducted. In addition, comparison of ANN-LM with other Al approaches illustrates the better accuracy and robustness of the ANN-LM model. However due to its intrinsic simplicity, the GMDH-NN models have also their own benefits. Altogether, the present study showed that the artificial intelligence approaches are promising tools to develop mathematical models to predict the performance and behavior of multisite catalysts for academic purposes, pilot plants, software and even industrial goals, though the results of such models are more reliable when experimental database is more complete.

\section{Compliance with ethical standards}

Conflict of interest The authors declare that they have no conflict of interest.

\section{References}

1. Kaminsky W (2008) Trends in polyolefin chemistry. Macromol Chem Phys 209:459-466

2. Vasile C (2000) Handbook of polyolefins, 2nd edn. Marcel Dekker, New York

3. Galli P, Vecellio G (2001) Technology: driving force behind innovation and growth of polyolefins. Prog Polym Sci 26:1287-1336

4. Alt HG, Köppl A (2000) Effect of the nature of metallocene complexes of group IV metals on their performance in catalytic ethylene and propylene polymerization. Chem Rev 100:1205-1221

5. Razavi A, Thewalt U (2006) Site selective ligand modification and tactic variation in polypropylene chains produced with metallocene catalysts. Coord Chem Rev 250:155-169

6. Resconi L, Cavallo L, Fait A, Piemontesi F (2000) Selectivity in propene polymerization with metallocene catalysts. Chem Rev 100:1253-1345

7. Sturzel M, Mihan S, Mulhaupt R (2016) From multisite polymerization catalysis to sustainable materials and all-polyolefin composites. Chem Rev 116:1398-1433

8. Hofmann D, Kurek A, Thomann R, Schwabe J, Mark S, Enders M, Hees T, Mülhaupt R (2017) Tailored nanostructured HDPE Wax/UHMWPE reactor blends as additives for melt-processable 
all-polyethylene composites and in situ UHMWPE fiber reinforcement. Macromolecules 50:8129-8139

9. Kurek A, Mark S, Enders M, Stürzel M, Mülhaupt R (2014) Two-site silica supported $\mathrm{Fe} / \mathrm{Cr}$ catalysts for tailoring bimodal polyethylenes with variable content of UHMWPE. J Mol Catal A: Chem 383-384:53-57

10. Kurek A, Xalter R, Stürzel M, Mülhaupt R (2013) Silica nanofoam (NF) supported single- and dual-site catalysts for ethylene polymerization with morphology control and tailored bimodal molar mass Distributions. Macromolecules 46:9197-9201

11. Kurek A, Mark S, Enders M, Kristen MO, Mülhaupt R (2010) Mesoporous silica supported multiple singleSite catalysts and polyethylene reactor blends with tailor-made trimodal and ultra-broad molecular weight distributions. Macromol Rapid Commun 31:1359-1363

12. Zhong F, Thomann R, Mülhaupt R (2019) Tailoring Mono-, Bi-, and trimodal molar mass distributions and all-hydrocarbon composites by ethylene polymerization on Bis(imino)pyridine Chromium(III) supported on ultrathin gibbsite single crystal nanoplatelets. Macromolecules 52:2701-2711

13. Stürzel $M$, Hees T, Enders $M$, Thomann $Y$, Blattmann $H$, Mülhaupt $\mathrm{R}$ (2016) Nanostructured polyethylene reactor blends with tailored trimodal molar mass distributions as melt-processable all-polymer composites. Macromolecules 49:8048-8060

14. Stürzel M, Kempe F, Thomann Y, Mark S, Enders M, Mülhaupt R (2012) Novel graphene UHMWPE nanocomposites prepared by polymerization filling using single-site catalysts supported on functionalized graphene nanosheet dispersions. Macromolecules 45:6878-6887

15. Stürzel M, Kurek AG, Hees T, Thomann Y, Blattmann H, Mülhaupt $\mathrm{R}$ (2016) Multisite catalyst mediated polymer nanostructure formation and self-reinforced polyethylene reactor blends with improved toughness/stiffness balance. Polym 102:112-118

16. Stürzel M, Thomann Y, Enders M, Mülhaupt R (2014) Graphenesupported dual-site catalysts for preparing self-reinforcing polyethylene reactor blends containing UHMWPE nanoplatelets and in situ UHMWPE shish-kebab nanofibers. Macromolecules 47:4979-4986

17. Zhong F, Schwabe J, Hofmann D et al (2018) All-polyethylene composites reinforced via extended-chain UHMWPE nanostructure formation during melt processing. Polym 140:107-116

18. Zhong F, Thomann R, Mülhaupt R (2018) Processing-nanostructure-property relationships of all-polyethylene composites reinforced by flow-induced oriented crystallization of UHMWPE. Macromol Mater Eng 1800022:1-8

19. Atashrouz S, Amini E, Pazuki G (2014) Modeling of surface tension for ionic liquids using group method of data handling. Ionics 21:1595-1603

20. Atashrouz S, Mirshekar H, Hemmati-Sarapardeh A (2017) A softcomputing technique for prediction of water activity in PEG solutions. Colloid Polym Sci 295:421-432

21. Atashrouz S, Mozaffarian M, Pazuki G (2015) Modeling the thermal conductivity of ionic liquids and ionanofluids based on a group method of data handling and modified maxwell model. Ind Eng Chem Res 54:8600-8610

22. Atashrouz S, Mozaffarian M, Pazuki G (2016) Viscosity and rheological properties of ethylene glycol + water $+\mathrm{Fe}_{3} \mathrm{O}_{4}$ nanofluids at various temperatures: experimental and thermodynamics modeling. Korean J Chem Eng 33:2522-2529

23. Atashrouz S, Pazuki G, Alimoradi Y (2014) Estimation of the viscosity of nine nanofluids using a hybrid GMDH-type neural network system. Fluid Phase Equilib 372:43-48

24. Dodangeh M, Gharanjig K, Arami M, Atashrouz S (2014) Surface alteration of polyamide fibers by polyamidoamine dendrimers and optimization of treatment process using neural network towards improving their dyeing properties. Dye Pigment 111:30-38

25. Shahriari S, Atashrouz S, Pazuki G (2018) Mathematical model of the phase diagrams of ionic liquids-based aqueous two-phase systems using the group method of data handling and artificial neural networks. Theor Found Chem Eng 52:146-155

26. Hashemkhani $M$, Soleimani R, Fazeli $H$, Lee $M$, Bahadori A, Tavalaeian M (2015) Prediction of the binary surface tension of mixtures containing ionic liquids using support vector machine algorithms. J Mol Liq 211:534-552

27. Hemmati-Sarapardeh A, Aminshahidy B, Pajouhandeh A, Yousefi SH, Hosseini-Kaldozakh SA (2016) A soft computing approach for the determination of crude oil viscosity: light and intermediate crude oil systems. J Taiwan Inst Chem Eng 59:1-10

28. Böhm LL (2003) The ethylene polymerization with Ziegler catalysts: fifty years after the discovery. Angew. Chemie Int. Ed. 42:5010-5030

29. Galli P, Vecellio G (2004) Polyolefins: the most promising largevolume materials for the 21st century. J Polym Sci, Part A: Polym Chem 42:396-415

30. Mülhaupt R (2003) Catalytic polymerization and post polymerization catalysis fifty years after the discovery of Ziegler's catalysts. Macromol Chem Phys 204:289-327

31. Ruff M, Paulik C (2012) Controlling polyolefin properties by inreactor blending, 1-polymerization process, precise kinetics, and molecular properties of UHMW-PE polymers. Macromol React Eng 6:302-317

32. Ruff M, Paulik C (2013) Controlling polyolefin properties by in-reactor blending: 2 particle design. Macromol React Eng 7:71-83

33. Severn JR, Chadwick JC, Duchateau R, Friederichs N (2005) "Bound but not gagged" immobilizing single-site a-olefin polymerization catalysts. Chem Rev 105:4073-4147

34. Stürzel M, Kurek A, Anselm M, Halbach T, Mülhaupt R (2013) Polyolefin nanocomposites and hybrid catalysts. In: Kaminsky W (ed) Polyolefins: 50 years after Ziegler and Natta II. Springer, Berlin, pp 279-309

35. Karimi H, Yousefi F (2007) Correlation of vapour liquid equilibria of binary mixtures using artificial neural networks. Chin J Chem Eng 15:765-771

36. Atashrouz $\mathrm{S}$, Mirshekar H (2014) Phase equilibrium modeling for binary systems containing $\mathrm{CO}_{2}$ using artificial neural networks. Bulg Chem Commun 46:104-116

37. Chakraborty M, Bhattacharya C, Dutta S (2003) Studies on the applicability of artificial neural network (ANN) in emulsion liquid membranes. J Memb Sci 220:155-164

38. Malallah A, Nashawi IS (2005) Estimating the fracture gradient coefficient using neural networks for a field in the Middle East. J Pet Sci Eng 49:193-211

39. Manohar HJ, Saravanan R, Renganarayanan S (2006) Modelling of steam fired double effect vapour absorption chiller using neural network. Energy Convers Manag 47:2202-2210

40. Hagan MT, Menhaj MB (1994) Training feedforward networks with the Marquardt algorithm. IEEE Trans Neural Netw 5:989-993

41. Kisi Ö, Uncuoguglu E (2005) Comparison of three back-propagation training algorithms for two case studies. Indian J Eng Mater Sci 12:434-442

42. Ameli F, Hemmati-Sarapardeh A, Schaffie $M$, Husein MM, Shamshirband $S$ (2018) Modeling interfacial tension in $N_{2} /$ n-alkane systems using corresponding state theory: application to gas injection processes. Fuel 222:779-791

43. Bose NK, Liang P (1996) Neural network fundamentals with graphs, algorithms, and applications. McGraw-Hill series in electrical computer engineering. McGraw-Hill, New York 
44. Zupan J, Gasteiger J (1993) Neural networks for chemists: an introduction. Wiley, Hoboken

45. Ivakhnenko AG (1968) The group method of data of handling; a rival of the method of stochastic approximation. Sov Autom Control 13:43-55

46. Ivakhnenko AG (1971) Polynomial theory of complex systems. IEEE Trans Syst Man Cybern SMC 1:364-378

47. Atashrouz S, Pazuki G, Kakhki SS (2015) A GMDH-type neural network for prediction of water activity in glycol and Poly(ethylene glycol) solutions. J Mol Liq 202:95-100

48. Suykens J, Gestel TV, Brabanter J (2002) Least squares support vector machines. World Scientific, Singapore

49. Suykens J, Vandewalle J (1999) Least squares support vector machine classifiers. Neural Process Lett 9:293-300

50. Xavier-De-Souza S, Suykens J, Vandewalle J, Bolle D (2010) Coupled simulated annealing. IEEE Trans Syst Man Cybern Part B Cybern 40:320-335

51. Atashrouz S, Mirshekar H, Hemmati-Sarapardeh A, Moraveji MK, Nasernejad B (2016) Implementation of soft computing approaches for prediction of physicochemical properties of ionic liquid mixtures. Korean J Chem Eng 32:1-15

52. Van Gestel T, Suykens J, Baesens B (2004) Benchmarking least squares support vector machines. Mach Learn 54:5-32

53. Paduszyński K, Domańska U (2014) Viscosity of ionic liquids: an extensive database and a new group contribution model based on a feed-forward artificial neural network. J Chem Inf Model 54:1311-1324

54. Hong Y, Fei L, Yuge X, Jin L (2008) GA Based LS-SVM classifier for waste water treatment process. In: 27th Chinese control conference

55. Gupta A, Ingle VR (2012) Optimization of LS-SVM parameters using genetic algorithm to improve DGA based fault classification of transformer-a review. Int J Sci Res Publ 2:1-4

56. Bastani D, Hamzehie ME, Davardoost F, Mazinani S, Poorbashiri A (2013) Prediction of $\mathrm{CO}_{2}$ loading capacity of chemical absorbents using a multi-layer perceptron neural network. Fluid Phase Equilib 354:6-11

57. Ketabchi S, Ghanadzadeh H, Ghanadzadeh A, Fallahi S, Ganji M (2010) Estimation of VLE of binary systems (tertbutanol + 2-ethyl-1-hexanol) and (n-butanol + 2-ethyl-1-hexanol) using GMDH-type neural network. J Chem Thermodyn 42:1352-1355
58. Atashrouz S, Hemmati Sarapardeh A, Mirshekar H, Nasernejad B, Keshavarz Moraveji M (2016) On the evaluation of thermal conductivity of ionic liquids: modeling and data assessment. J Mol Liq 224:648-656

59. Hemmati-Sarapardeh A, Ameli F, Varamesh A, Shamshirband S, Mohammadi AH, Dabir B (2018) Toward generalized models for estimating molecular weights and acentric factors of pure chemical compounds. Int J Hydrogen Energy 43:2699-2717

60. Hemmati-Sarapardeh A, Varamesh A, Husein MM, Karan K (2018) On the evaluation of the viscosity of nanofluid systems: modeling and data assessment. Renew Sustain Energy Rev 81:313-329

61. Leroy AA, Rousseeuw PJ (1987) Robust regression and outlier detection. Wiley series in probability and mathematical statistics. Wiley, New York

62. Hosseinzadeh M, Hemmati-Sarapardeh A (2014) Toward a predictive model for estimating viscosity of ternary mixtures containing ionic liquids. J Mol Liq 200:340-348

63. Mulhaupt R, Kurek A, Sturzel M, Zhong F, Hees T (2018) All polyolefin composites via nanostructure formation during melt processing. In: 3rd edition of international conference and exhibition on polymer chemistry, March 26-28, Austria

64. Sieb D, Baker RW, Wadepohl H, Enders M (2012) Naphthyridine cyclopentadienyl chromium complexes as single-site catalysts for the formation of ultrahigh molecular weight polyethylene. Organometallics 31:7368-7374

65. Mark S, Kurek A, Mülhaupt R et al (2010) Hydridoboranes as modifiers for single-site organochromium catalysts: from lowto ultrahigh-molecular-weight polyethylene. Angew Chemie Int Ed 49:8751-8754

66. Yildiz Z, Uzun H (2015) Prediction of gas storage capacities in metal organic frameworks using artificial neural network. Microporous Mesoporous Mater 208:50-54

67. Anantawaraskul S, Toungsetwut M, Pinyapong R (2008) Determination of operating conditions of ethylene/1-octene copolymerization using artificial neural network (ANN). Macromol Symposia 264:157-162

Publisher's Note Springer Nature remains neutral with regard to jurisdictional claims in published maps and institutional affiliations. 